Archives de sciences sociales des religions

180 | octobre-décembre 2017

Bulletin bibliographique

\title{
Le Centre Thomas More : genèse et enjeux
}

\section{Tangi Cavalin}

\section{(2) OpenEdition}

Journals

Édition électronique

URL : https://journals.openedition.org/assr/29717

DOI : $10.4000 /$ assr. 29717

ISSN : 1777-5825

Éditeur

Éditions de l'EHESS

Édition imprimée

Date de publication : 1 décembre 2017

Pagination : 19-34

ISSN : 0335-5985

\section{Référence électronique}

Tangi Cavalin, «Le Centre Thomas More : genèse et enjeux », Archives de sciences sociales des religions [En ligne], 180 | octobre-décembre 2017, mis en ligne le 01 décembre 2019, consulté le 22 septembre 2021. URL : http://journals.openedition.org/assr/29717 ; DOI : https://doi.org/10.4000/assr.29717 


\section{Tangi Cavalin}

\section{Le Centre Thomas More : genèse et enjeux}

À l'instar de celle de la Province dominicaine de Lyon dont il est une création, l'histoire du Centre Thomas More est mal connue. Fondé en $1970^{1}$, il a son siège au couvent Sainte-Marie édifié par Le Corbusier au domaine de la Tourette, situé sur la commune d'Éveux dans les Monts du Lyonnais. Il organise pendant une trentaine d'années des sessions de sciences humaines qui rassemblent chaque année des centaines de participants ${ }^{2}$, avant de céder la place, en 2002, au Centre culturel de rencontres de la Tourette ${ }^{3}$. Si quelques rares travaux pionniers éclairent telle ou telle facette de sa trajectoire, travaux dus pour l'essentiel à ses acteurs dominicains, l'histoire du Centre Thomas More, lieu de rencontres et d'échanges sans réel équivalent en France, reste à écrire. Cette étude n'est qu'une contribution modeste à cette histoire, qui entend surtout soumettre à l'examen les quelques textes, édités ou non, qui retracent ses débuts et les confronter aux archives dominicaines afin de restituer le terreau dans lequel s'enracine cette fondation. Il semble acquis, en effet, que la naissance du Centre et l'inclusion dans son champ d'attribution des seules sciences humaines - il faudrait presque dire, pour les premières sessions, de la seule sociologie des religions - entérineraient un bouleversement dans la hiérarchie des savoirs, bouleversement d'autant plus spectaculaire qu'il s'inscrirait dans un bâtiment dédié jusqu'alors à la formation théologique de jeunes religieux

1. La première session du Centre s'ouvre en mai 1970. À cette date, il ne possède pas de réalité juridique. La création officielle du Centre Thomas More sous la forme d'une association régie par la loi de 1901 a lieu lors d'une assemblée constitutive tenue à la Tourette le 17 avril 1972; l'annonce est publiée au Journal Officiel du 22 octobre 1972.

2. De 1970 à 1990, selon les chiffres établis par André Laudouze, 329 sessions ont rassemblé près de 12000 participants. Les thèmes des sessions portent fréquemment sur des sujets religieux, sans pour autant que ces dernières soient majoritaires. Les disciplines concernées relèvent d'abord de la sociologie des religions, mais aussi d'autres champs de la sociologie, de l'histoire, de la psychanalyse, de la linguistique, de l'ethnologie, de l'anthropologie ou encore du travail social et des sciences de l'éducation (Laudouze, 1990: 37-38).

3. Le Centre culturel naît de la fusion de deux associations, celle du Centre Thomas More et celle de l'Accueil dominicain. Placée en redressement judiciaire en 2008, l'association gérant le Centre culturel est mise en liquidation judiciaire par le Tribunal de grande instance de Lyon le 26 mai 2009. 
et qu'il serait le fait de leurs formateurs ecclésiastiques ${ }^{4}$. La théologie serait, dans cette opération, exclue du champ des savoirs, y compris des savoirs religieux au profit des seules sciences humaines et sociales triomphantes au cours de ces années: après avoir été la «reine des sciences », l'organisatrice de l'ordre des savoirs, elle serait réduite au terme d'un singulier retournement à n'être plus qu'un objet d'étude pour les sciences humaines. Le Centre Thomas More, plus encore que les autres centres de formation abrités sur le domaine de la Tourette dans les années 1960-19805 , serait ainsi, avec Boquen en Bretagne et quelques autres lieux d'Église (Boitel, 1975 ; Lebel, 2015), un foyer privilégié de la sécularisation et de la contestation qui caractérisent alors la pensée catholique française et européenne (Pelletier, Schlegel, 2012). Il participerait d'une rupture de transmission de la théologie comme discipline entre les générations dominicaines ${ }^{6}$. Replacée dans une temporalité plus longue afin de contourner la difficulté que représente la politisation des enjeux intellectuels dans les années 1968, l'apparente désertion de la théologie au profit des sciences humaines pourrait se lire comme un nouvel avatar du conflit entre la pensée chrétienne et la recherche scientifique au cœur de la crise moderniste. Les sciences humaines occuperaient désormais la place exercée dans les années 1900 principalement par l'histoire et, dans une moindre mesure, par la jeune sociologie durkheimienne. D'un moment intellectuel à l'autre, le même relativisme est à l'œuvre qui sape la revendication de transcendance de la théologie. Peut-on, au-delà de ces approches éprouvées, se risquer à une autre esquisse des raisons de la fondation du Centre Thomas More, en empruntant un chemin inverse à celui précédemment évoqué, c'est-à-dire en s'arrêtant sur le temps court et sur une histoire interne, au risque assumé de la myopie? Le détour par une histoire-bataille de la genèse du Centre Thomas More est-il de nature à infléchir le regard porté sur cette création? À se tenir sur cette ligne interprétative, sans pour autant rejeter les approches en termes de sécularisation et de conflit entre régimes de vérité opposés, il semble possible d'étayer l'hypothèse selon laquelle les fondateurs dominicains du Centre Thomas More, manifestant dans la crise un parfait esprit de corps professoral et religieux, se sont employés à préserver l'espace intellectuel de la théologie plutôt qu'à lui substituer, sans transition et sans compromis, un terrain de rencontres entièrement occupé par les sciences humaines et sociales.

4. Selon son secrétaire général, Roland Ducret, «entre autres refus, le Centre Thomas More veut donc ignorer ce que la foi chrétienne nous enseigne de l'homme, même si, par la force de l'histoire de l'Occident, il ne peut se soustraire complètement aux perspectives du christianisme dans lequel l'Occident est né " (Ducret, 1984: 9).

5. Le Centre Saint-Dominique, qui lui préexiste - avec une interruption liée à l'installation provisoire du studium dans ses locaux - depuis les années 1950, et le Centre Albert-le-Grand, qui tient sa première réunion quelques mois après les débuts du Centre Thomas More, en octobre 1970, dans une perspective différente.

6. C'est la thèse défendue de manière précoce et argumentée, sur le terrain universitaire, par Gérard Cholvy et Yves-Marie Hilaire (Cholvy, Hilaire, 1988: 312). 


\section{Une mémoire archétypale de la genèse du Centre Thomas More}

Pour étayer cette proposition liminaire, il convient de rappeler brièvement, avant de les questionner, les principaux traits qui caractérisent la fondation du Centre sur le domaine de la Tourette. L'obstacle à surmonter auparavant est d'abord celui d'une production historiographique lacunaire: à la suite d'Étienne Fouilloux, il faut faire le constat d'une Province de Lyon délaissée par les historiens, comparativement à ses voisines de France et de Toulouse (Donneaud, Laffay, Montagnes, 2015 : 5). Sa naissance est le fruit d'un conflit interne à la Province de France restaurée par Henri-Dominique Lacordaire, conflit qui se solde par la création dissidente d'un couvent à Lyon en 1856 (Cavalin, Viet-Depaule, 2015) puis d'une province en 1862: sous couvert de retour aux observances voulues par les fondateurs médiévaux de l'Ordre, la nouvelle province se raccroche à la matrice de l'intransigeantisme en cours de définition sous le pontificat de Pie IX. Certaines des initiatives qui jalonnent l'histoire de la Province de Lyon au $\mathrm{Xx}^{\mathrm{e}}$ siècle ont certes attiré l'attention, comme celle d'Économie et Humanisme (Pelletier, 1996) ou de Jeunesse de l'Église et de son fondateur Maurice Montuclard (Ramognino, 1991; Keck, 2004; Keck, 2015), et quelques-unes de ses figures marquantes de l'après-guerre sont depuis peu mieux connues comme celles de François Biot (Rousseau, 2015), d'Henri-Charles Chéry (Chantin, 2015) ou de Gabriel Turin (Chatelan, 2015). Sa tradition intellectuelle néanmoins, si l'on excepte sa revue Lumière \& Vie active de 1951 à 2013 à partir du foyer lyonnais et non du studium de la Tourette (Fouilloux, 2015), reste dans l'ombre. Surtout, le poids des origines intransigeantes de la Province reste d'autant plus difficile à évaluer que les travaux mentionnés ci-dessus ont pour point commun d'attirer l'attention sur les créations les plus novatrices, voire les plus progressistes, de son histoire qui, comble du paradoxe, n'en manque pas. Quant aux années 1968 de la province, elles constituent un véritable angle mort et souffrent de la comparaison avec les études menées sur les provinces de France et de Toulouse (Raison du Cleuziou, 2016; Donneaud, Laffay, Montagnes, 2015) ou encore sur les jésuites peut-être mieux armés que les dominicains à relever le défi intellectuel posé par les sciences humaines (Fouilloux, Gugelot, 2014). Un tel bilan historiographique incite à la prudence dans une analyse des débuts du Centre Thomas More qui ne peut, en attendant d'autres investigations, faire l'économie d'un retour sur les faits.

Sous bénéfice d'inventaire, quelques jalons peuvent être proposés sur la base des témoignages des acteurs: l'impulsion initiale est à chercher dans l'introduction subite, au cœur du studium de la Province de Lyon, d'une contestation massive en Mai 1968. Elle provoque une déstabilisation si forte qu'il perd sa raison d'être comme lieu de formation des novices dominicains. Privé de ses étudiants partis pour Lyon avec quelques-uns de leurs formateurs, le couvent Sainte-Marie, œuvre de l'architecte Le Corbusier inaugurée moins de dix ans auparavant, en octobre 1960, n'est pas pour autant abandonné. Le corps de lecteurs reste pour l'essentiel soudé autour de la conviction qu'il est possible d'investir le lieu d'une manière inédite. Le studium est alors un lieu en attente de reconversion, à la différence de ce qui se passe au cours de la même séquence dans la Province de France où il est décidé de transférer les études du couvent 
d'Étiolles à Paris 7 . Un témoin privilégié de ces années tournantes, Roland Ducret, professeur de philosophie au couvent, mais aussi vicaire du prieur Paul Grandin puis secrétaire général du Centre Thomas More de 1978 à 1990, a fourni de ces débuts un récit au style tout entier emprunt de déterminisme:

Le Centre Thomas More fut projeté et réalisé dans les deux années 1969-1970. Les instigateurs et pionniers de cette entreprise furent Henri Desroche et Paul Gran$\operatorname{din}^{8}$. [...] À l'heure du désarroi créé à la Tourette, en 1969-1970, par la diminution importante des effectifs des jeunes frères étudiants dominicains, la dispersion en d'autres communautés d'une partie de ceux qui restaient et la suppression du statut de couvent d'études pour le beau bâtiment [...] créé par Le Corbusier en 1960, le père Paul Grandin ne put que prendre le chemin de l'avenue Franco-Russe 9 . (Ducret, 1997: 41)

André Laudouze, également dominicain de la Province de Lyon et acteur de cette histoire comme secrétaire du Centre, dans la rétrospective la plus complète qui soit de ses deux premières décennies, avalise globalement ce caractère d'inévitabilité quelque peu providentiel, quoiqu'avec des nuances (Laudouze, 1990). Prenant appui sur le témoignage de Ducret ${ }^{10}$, il débute sa propre présentation en ces termes:

La décision de lancement a été prise au colloque tenu à Albiez-le-Vieux en Maurienne les 28, 29, 30 janvier 1970, à l'initiative de Paul Grandin, alors prieur du couvent Sainte-Marie de la Tourette (couvent dit «Le Corbusier») et d'Henri Desroche, alors directeur à la sixième section de l'École Pratique des Hautes Études, qui en avait émis l'idée lors d'un week-end à La Tourette en novembre 1969. (Laudouze, 1990: 7)

La fondation du Centre Thomas More a donc pour origine l'action opportune de quelques amis choisis (Grandin, Desroche) ${ }^{11}$, à l'issue de deux rencontres fondatrices aisément repérables dans le temps et dans l'espace (la Tourette chez les dominicains et surtout, en petit comité, au chalet d'Albiez-le-Vieux en Savoie).

7. Le chapitre provincial de 1969 en prend acte dans ses décisions pratiques: «Avenir de la Tourette. La question a été exposée au Chapitre et reprise par le Définitoire qui a donné son accord pour entreprendre la reconversion du couvent Sainte-Marie.» (B 1404, Chapitres provinciaux, 1969) 8. Paul Grandin (1913-1991), entré chez les frères prêcheurs en 1931, et Henri Desroche, vêtu de l'habit dominicain en 1933, appartiennent tous les deux à la même génération de dominicains de la Province de Lyon. Leur amitié date des années de studentat. Il faut noter pour notre propos que Grandin, en 1969-1970, n'est pas un novice en matière de sociologie religieuse, si ce n'est de sociologie des religions: comme aumônier (régional puis national) de la Jeunesse agricole catholique (JAC), puis comme acteur des missions régionales rurales dans les années 1950, il s'est familiarisé avec la sociologie du Chanoine Fernand Boulard comme avec celle pratiquée à Économie et Humanisme.

9. Le 7, avenue Franco-Russe à Paris est l'adresse du Collège Coopératif où Desroche avait un bureau.

10. "Aux dires de Roland Ducret, "le centre est né de l'amitié de Paul Grandin et Henri Desroche” » (Laudouze, 1990: 9).

11. Le texte d'Henri Desroche, "Avec le Centre Thomas More. Pour son vingtième anniversaire ", partiellement cité par Roland Ducret et, à sa suite, par André Mary, confirme, de mémoire, les grandes lignes de ce récit (Ducret, 1997: 42-43; Mary, 2008). Une copie de ce texte est conservée aux ADPL B 1375. Les Mémoires d'un faiseur de livres n'apportent guère plus de précisions (Desroche, 1992). 
Une mémoire s'est constituée, très tôt semble-t-il ${ }^{12}$, insistant sur l'importance de la rencontre d'Albiez au cours de laquelle les présents se reconnaissent dans le patronage de Thomas More et sur l'entrée en scène décisive d'un Henri Desroche comme préparé à cette fonction par son propre itinéraire personnel chez les dominicains de la Province de Lyon (1933-1950), par son rôle de fondateur du Groupe de sociologie des religions au milieu des années 1950, par son intérêt pour les questions de développement qui seront rapidement très présentes dans les sessions du Centre, et encore par son goût pour les utopies concrètes.

\section{Du studium au campus de la Tourette}

Sans vouloir en rien minimiser le rôle des acteurs, à coup sûr déterminant lorsque l'on se situe dans le rythme de l'événementiel, il faut relever, dans ces retours sur les débuts du Centre, d'une part une absence de souci d'intégration dans un contexte particulier, si ce n'est celui, jamais circonscrit dans ses effets ni caractérisé dans ses formes spécifiques, de Mai 68, d'autre part un oubli de tout ce qui pourrait faire de cette action une expérience élaborée avec d'autres dans un processus plus complexe que ne le laisse suggérer la réduction à un événement inaugural, celui d'Albiez-le-Vieux en janvier 1970.

La consultation des archives dominicaines ${ }^{13}$ plaide en faveur d'un élargissement de la focale en amont de cette date et au-delà des deux fondateurs reconnus. Du côté des dominicains, pour s'en tenir à ce seul versant, il est clair que c'est un studium globalement uni autour de Paul Grandin qui mène une réflexion collective au cours de la phase préparatoire. Trois séries de remarques peuvent être avancées pour caractériser la nouvelle donne de ces années 1968-1970.

En premier lieu, le cercle de ceux qui participent aux réunions qui préparent la naissance du Centre à partir de l'automne 1969 ne correspond pas à strictement parler au corps des lecteurs du studium (soit, en 1970, Bruno Carra de Vaux, Hilaire Prisset, François Berrouard, Joseph Lemarchand, François Biot, Cyrille Ducret, François Genuyt, Yves-Bernard Trémel, Jean-Claude Sagne). Le premier des centres de la Tourette, le Centre Saint-Dominique, dont l'autonomie à l'égard du couvent d'études est soigneusement préservée, a néanmoins fourni son expertise en matière d'animation de sessions avec des interventions de ses deux maîtres d'œuvre, Jean-Pierre Lintanf et François Nielly. Un coopérateur, Marcel Veillet, avant de quitter l'Ordre peu après, participe aux premières réunions. Pierre Cren, également présent au cours de cette phase, s'il a été lecteur au couvent Sainte-Marie, a rejoint en 1969 la nouvelle communauté des étudiants de la rue Montagny à Lyon tout en restant lié à la réflexion poursuivie à Éveux. Les consultations et les échanges, au sein de

12. La lecture du Bulletin du Centre Thomas More en fournit une illustration au moins dès le liminaire de sa première livraison, en mars 1973, qui fixe la date de naissance: «Depuis le mois de janvier 1970, date de sa fondation, le Centre Thomas More a consacré ses efforts à vivre et à se développer. » Cette mémoire des fondations est par la suite constamment reprise jusque dans les termes employés.

13. Nous avons utilisé les archives conservées à Paris (Saulchoir), dans la section réservée aux archives dominicaines de la Province de Lyon (ADPL) sous la cote B 1534, ainsi que celles du couvent de la Tourette. L'enquête repose également sur la reconstitution des itinéraires des frères de la Province de Lyon présents sur le domaine de la Tourette au cours de ces années. 
la communauté dominicaine, ont lieu sans exclusive: Vincent Cosmao, directeur adjoint de l'IRFED après la mort du père Lebret, qui se dit intéressé par le projet lors d'une rencontre de novembre 1969 avec Grandin, y apporte son concours; Alain-Zacharie Serrand, venu des Éditions du Cerf et, entre autres assignations, de l'aumônerie des Dominicaines des campagnes, ayant manifesté son intérêt, participe à la session d'Albiez-le-Vieux et rejoint le Centre Thomas More en gestation en février 1970; Antoine Lion, jeune dominicain de la Province de France, est également au nombre des participants à la session savoyarde de janvier 1970 et occupera une place de choix dans l'histoire du Centre. Autour d'un noyau actif de lecteurs, lui-même animé par Paul Grandin, gravitent ainsi des personnalités disponibles pour la rencontre des frères prêcheurs avec les sciences humaines.

Une seconde série de remarques se dégage à l'examen des archives, quant à la contribution proprement dominicaine de la genèse du Centre Thomas More: de manière plus nuancée que ce que donnent à lire les récits d'anamnèse évoqués plus haut, la part de l'élaboration dominicaine doit être réévaluée à la hausse de manière à éviter de faire d'Henri Desroche l'instigateur d'une réflexion qui se cherche en amont, tout au long de la fin de l'année 1968 et de l'année 1969, au fil des réunions, des échanges entre religieux, des visites aux personnalités ecclésiastiques comme à l'ensemble des chercheurs du Groupe de sociologie des religions. Peut-il en être autrement d'ailleurs? Le mode de gouvernement des institutions dominicaines qui donne une large place aux échanges, aux consultations, aux votes à tous les échelons, mode de gouvernement encore accentué par la multiplication des groupes de recherche et l'incitation à la formation permanente au cours des années 1968, doit incliner, pour le moins, à considérer avec prudence la génération pour ainsi dire spontanée d'un Centre de rencontres dédié aux sciences humaines. Les dominicains de la Province de Lyon, comme l'ensemble des dominicains français, mais de manière peut-être plus vive, partagent assez largement le sentiment que l'enseignement reçu au cours des années de studentat (philosophie et théologie) se révèle insuffisant pour mener à bien leur mission de frères prêcheurs dans une société française en évolution constante. L'idée d'un nécessaire recyclage pour les prêtres est ainsi au cœur de la démarche du Centre Saint-Dominique depuis le tournant des années 1960, le public visé étant, en l'espèce, celui d'ecclésiastiques ayant souvent vécu hors de France, dans les missions, et se situant de ce fait en porte-à-faux avec la culture catholique et la société française au fur et à mesure que les indépendances des anciennes colonies conduisent certains d'entre eux à revenir en France. Mais cette nécessité d'une mise à niveau n'est pas restée l'apanage des missionnaires: en dépit d'un long cycle d'études initial de sept années ${ }^{14}$, il est admis dans les rangs dominicains, à tous les échelons si ce n'est unanimement, qu'elle vaut aussi pour les frères prêcheurs dont la vocation exige, plus encore que pour d'autres religieux, qu'ils soient au fait de la culture de leur temps, en particulier de celle qui était produite par les milieux universitaires et la recherche comme le rappellent avec constance les chapitres provinciaux. Du 18 août au 13 septembre 1968, sous la direction d'un triumvirat

14. Le chapitre provincial de 1967 fixe à sept années le nombre d'années d'études, réparties en deux cycles: le premier procure en cinq ans la formation commune en philosophie et théologie avant qu'un second cycle de deux années n'amène les étudiants à se spécialiser en fonction de leurs affinités et des besoins de la Province. 
constitué des pères Paul Grandin pour la Province de Lyon, Claude Bigard pour la Province de France et Augustin Souques pour la Province de Toulouse, se tient ainsi au grand séminaire de Saint-Flour un stage interprovincial de recyclage pour dominicains quarantenaires qui ne doit pas grand-chose aux événements du printemps précédent puisque son organisation relève d'une décision prise lors des chapitres provinciaux de $1967^{15}$.

Au cours des deux années qui précèdent l'ouverture du Centre Thomas More, ce qui se donne à voir dans la Province de Lyon, c'est une tendance à doubler les instances constitutionnelles par une sociabilité plus spontanée faite de comités ou de rencontres temporaires, née en fonction des nécessités ressenties et, parfois, des affinités personnelles. Nulle intention de subversion des institutions traditionnelles dans cette évolution que ces dernières avalisent et suscitent même, mais plutôt celle de multiplier lieux et moments de réflexion selon des formes souples adaptées aux besoins d'une période de crise. La tenue d'assises provinciales exceptionnelles, ouvertes à tous les frères en exprimant le souhait, début juillet 1969, dans les jours qui précèdent la tenue d'un chapitre provincial à Eveux, est exemplaire de ce fonctionnement. C'est selon la même logique que se met sur pied, à la fin septembre 1969, un comité composé pour l'essentiel des lecteurs du studium autour du prieur Paul Grandin qui se réunit sur un rythme hebdomadaire jusqu'en juin 1970, c'està-dire jusqu'à la première session du Centre Thomas More dont ce comité est l'organisateur immédiat ${ }^{16}$. C'est en son sein que sont discutées les options envisagées et que se formalise peu à peu, aux côtés d'autres initiatives, le projet d'espace dédié aux sciences humaines. Plusieurs de ses participants prennent successivement le chemin de Paris auprès des collaborateurs scientifiques pressentis du Groupe de sociologie des religions, Desroche, Séguy, Poulat, Maître en particulier. Ils n’y sont d'ailleurs pas toujours reçus de manière encourageante, comme le suggère le récit de l'entretien entre Paul Grandin et Émile Poulat rapporté par le premier aux autres membres du comité le 3 novembre: «[Poulat] estime qu'on a peu de chances de réussir: "vous restez trop philosophes et théologiens". Nous voit obligés de nous ressourcer (sciences humaines). É. Poulat est un scientifique, homme d'une grande rigueur intellectuelle [...]. Après cet entretien, on peut mesurer les risques et les chances de notre entreprise. » Si ces rencontres parisiennes viennent nourrir les réflexions du comité, celui-ci ne les a pas attendues pour élaborer ses propres bases de discussion. Paul Grandin, prieur de la communauté, Bernard Nielly, lecteur et ancien provincial, et François Genuyt, régent des études, sont ainsi les auteurs de rapports entre dominicains avant d'être soumis aux invités du Groupe de sociologie des religions, Desroche en tête, lors d'une première rencontre à la Tourette, le 22 novembre 1969.

15. Ce type de session, d'au moins un mois, est conçu pour être périodique selon la décision des définitoires des trois provinces dominicaines rapportée dans les Actes du chapitre de la Province de Lyon de mai-juin 1967. Il n'est d'ailleurs que l'un des volets d'une ambitieuse politique de formation et de développement de la recherche scientifique déjà promue à Paris par le Centre international de Recherche et d'Échanges culturels (CIREC) et fortement approuvé lors du chapitre général de l'Ordre des frères prêcheurs tenu à Bogotá (Colombie) en juillet 1965 (B 1404, Chapitres provinciaux, 1967). Les ambitions affichées devront souvent être revues à la baisse, mais l'exigence d'une vocation dominicaine à l'étude soulignée au chapitre de Bogotá restera une référence dans l'Ordre. 16. Les minutes de ces réunions sont rangées sous la cote ADPL, B 1375. 
Un troisième ensemble de considérations doit être assorti aux précédentes pour situer la genèse dominicaine du Centre Thomas More: il n'a jamais été question, dans la pensée de ses responsables, de substituer au studium un lieu de rencontres entièrement dévolu aux sciences humaines des religions. Dès les premières discussions qui suivent les soubresauts de Mai 1968 et après que la décision d'assurer désormais la formation des étudiants lyonnais ait été entérinée, une série d'options est envisagée par le corps des lecteurs décidé à maintenir sa présence au couvent Sainte-Marie. Ces options sont présentées comme complémentaires, relevant d'une forme de division du travail, plutôt que comme concurrentes. L'idée et l'expression d'un «campus de la Tourette» sur lequel se déploieraient des centres spécialisés émergent, semblet-il, dès l'été 1968. Elle n'est pas neuve si l'on n'omet pas de rappeler l'ancrage du Centre Saint-Dominique sur le domaine et son animation par Jean-Pierre Lintanf depuis 1959. Un texte programmatique établi par Paul Grandin après consultation des lecteurs et du provincial, Damase Belaud, dont la première version daterait de juin-juillet $1968^{17}$, formule ainsi quatre pistes à envisager pour l'animation de ce futur campus: "centre de pastorale supérieure du clergé", "centre spirituel d'expérimentation communautaire et liturgique», "centre de colloques théologiques avec participation multidisciplinaire ", "centre de recherche des sciences humaines de la religion». Il est précisé en ouverture de ce document que ces objectifs «ne sont pas nécessairement liés, mais [...] ne sont pas contradictoires ». Certaines des pistes explorées ne pourront finalement voir le jour. Ainsi, la première formulée, qui a pourtant toute la faveur de Grandin ${ }^{18}$, se révèle impossible à mettre en œuvre en raison du risque de concurrence avec d'autres centres de recyclage dans le diocèse de Lyon et dans la région apostolique. De même, l'idée d'un «centre spirituel d'expérimentation communautaire et liturgique ", quelque peu inspiré de l'expérience menée alors au Centre de la Sainte-Baume en Provence, pour lequel des contacts sont pris avec des communautés de religieuses pressenties pour en assurer l'animation, est finalement jugée irréalisable sous cette forme. Cependant, le Centre Albert-le-Grand, dont la cheville ouvrière est François Biot, reprendra et développera sous une autre forme cette intuition. Quant au projet sans doute le plus extérieur à la communauté dominicaine car inspiré des conseils enthousiastes de Mgr Pierre Haubtmann, recteur de l'Institut catholique de Paris, celui d'un «centre de colloques théologiques avec participation multidisciplinaire » destiné à offrir un cadre à la confrontation entre théologiens et spécialistes des sciences humaines, il n'est pas retenu.

Ce foisonnement de projets, progressivement mis en œuvre au cours des rencontres hebdomadaires de l'automne 1969, illustre suffisamment l'implication des acteurs dominicains dans la genèse du Centre Thomas More, comme leur souci de ne pas s'en tenir à une seule fondation, mais au contraire de faire de la Tourette un «lieu d'Église» où puisse se réinventer la tradition de recherche intellectuelle et de prédication de l'Ordre des frères prêcheurs.

17. Le contenu de ce texte de quatre pages permet de situer son élaboration entre juin 1968, date probable de son premier état, et mai 1969, moment de sa stabilisation, soit avant les Assises provinciales qui s'ouvrent le 29 juin. Il est, de fait, le texte de référence lors des discussions et rencontres de l'automne 1969 (ADPL, B 1375).

18. Selon les témoignages de Régis Morelon, alors étudiant (Le Saulchoir, 22 septembre 2016), et de Bruno Carra de Vaux, alors lecteur du studium (Saint-Nom de Jésus, 23 octobre 2016). 


\section{La genèse du Centre Thomas More au prisme de l'identité provinciale}

Au-delà des débats internes au petit groupe de religieux à l'origine, directe et indirecte, d'un «centre de recherche des sciences humaines de la religion", se pose la question de la réception de cette initiative dans la Province de Lyon. Le texte élaboré par Grandin et les lecteurs de la Tourette en 1968-1969 ne dissimule pas le caractère potentiellement subversif de cette option: elle est "pour la plupart des frères intéressés [...] la plus radicale, celle qui permettra une mutation en profondeur, et un renouvellement de l'intérêt de la recherche commune par la nouveauté du projet [...]. Par ailleurs, la réalisation de ce projet exigerait de la plupart des lecteurs intéressés, une "reconversion" vers une science humaine, en accord avec ses goûts et ses moyens, ayant certaine connaturalité avec sa spécialité théologique ». Malgré l'énoncé de ces risques et de ces exigences, c'est cette ligne d'orientation qui est privilégiée sans soulever, pour autant que la documentation permette d'en juger, d'objection majeure ${ }^{19}$. L'accueil fait par Desroche à cette intuition y est certainement pour beaucoup, d'autant qu'il est assorti de l'engagement simultané des autres membres du Groupe de sociologie des religions. La question de son acceptation parmi les principaux intéressés, les dominicains de la Province de Lyon, n'est pas résolue pour autant.

Un premier type de réponse repose sur l'hypothèse d'une fondation voulue par le seul corps professoral, sans que le reste de la province soit impliqué. Les archives confirment sur ce point la mémoire des acteurs d'un centre de rencontres voulu par le prieur et les lecteurs avec le soutien du provincial sans qu'il fasse l'objet d'une large consultation dans la province. Comment interpréter cette apparente indifférence? S'agit-il d'un manque d'intérêt, voire d'une défiance, pour un projet qui signe la fin du couvent d'études dans sa forme traditionnelle? Ou au contraire d'un blanc-seing accordé par le reste de la province aux recherches des lecteurs du studium, dans un strict respect des charges et des responsabilités dévolues à chacun?

\section{Les assises de la Tourette en 1969}

Quelques éléments de réponse sont à glaner dans les rapports des assises provinciales organisées dans la première semaine de juillet 1969 à la Tourette ${ }^{20}$. Ces assises sont, comme dans les autres provinces dominicaines de France, la conséquence immédiate des remises en cause de Mai 1968 et se donnent pour objectif de réfléchir, à la lumière des événements et des revendications des jeunes générations,

19. Les lecteurs ayant vécu le plus douloureusement la mise en cause estudiantine du mois de mai 1968 comme René-Antoine Gauthier et surtout Sébastien Lecapitaine ont quitté le couvent d'études à la faveur des vacances d'été. Ceux qui restent forment un groupe globalement soudé dans l'intention de réussir la reconversion du couvent d'études.

20. Les assises s'achèvent le 6 juillet, juste avant l'ouverture, le lendemain, dans le même lieu, du chapitre provincial qui entérine, sans y être en rien obligé d'un point de vue constitutionnel, la plupart des votes de ces assises. La documentation relative aux assises est classée sous la cote B 1132. Le fonds conventuel de la Tourette conserve un carton (non coté) «Assises de la Tourette, Province de Lyon, 1969 » complémentaire de ceux conservés dans les Archives provinciales. 
à différents thèmes de la vie dominicaine: «nouvelles formes de sociabilité dans l'Ordre », "partage de l'autorité », «renouveau liturgique », "rapport entre le dedans et le dehors », " place et statut de la théologie », «le travail professionnel », «la place de la politique », etc. Le document dix-neuf, intitulé «Projet pour l'utilisation du couvent de La Tourette », met à la disposition des participants aux assises la réflexion menée par les lecteurs du couvent Saint-Marie d'Éveux et rédigée par Paul Grandin. Un autre texte soumis aux sessionnaires, sous le titre «Le studium et son avenir...", envisage la question du point de vue du couvent d'études et du corps des lecteurs et non plus à partir du couvent dessiné par Le Corbusier. La liste des signataires - Paul Grandin, Bernard Nielly, Charles Noiray, Roland Ducret, Jean-Claude Sagne, Hugues Cousin, Noël Rondeau - recoupe partiellement le document précédent. Il prévoit la possibilité de maintenir les lecteurs à la Tourette ou, au contraire, d'opérer le transfert du corps des professeurs dans le couvent lyonnais du Saint-Nom de Jésus, en vue de la création d'un centre de réflexion théologique urbain. En dépit de la provocation aux échanges que constituent ces textes, avec bien d'autres il est vrai, ni les assises, ni le chapitre provincial qui se tient dans la foulée n'accordent d'importance à cette question. Le père Damase Belaud, provincial, reconnaît d'ailleurs, dans une lettre ouverte aux frères de la province rédigée à la date du 11 août $1969^{21}$, que les débats de l'été n'ont pas été polarisés par les questions liées au studium et à la formation des étudiants, comme cela a été le cas dans la Province de France avec le choix fait de quitter le Saulchoir d'Étiolles, mais par les communautés, nouvelles ou anciennes, et le type de vie dominicaine dont elles assurent la promotion. La reconversion du couvent d'Éveux, avalisée dans les actes du chapitre, n'a en rien constitué alors un enjeu de débat.

Le père Claude Pont, maître des novices puis maître des étudiants, figure incontournable de cette période, a fourni un récit de ces assises dont il est avec Paul Grandin une des chevilles ouvrières (Pont, 2010). Il y voit un moment essentiel de la reformulation de la vocation dominicaine dans la crise de l'après-68. Il note que tous les textes, même les plus novateurs, ont été votés à une écrasante majorité des présents (autour de 120-125 votes favorables pour 135 présents ou votants environ, ce que confirment les archives) et s'interroge sur l'expression de cette confiance. Les présents ont-ils mesuré la portée des textes auxquels ils offraient leur approbation? Ont-ils cru qu'ils ne les engageaient pas puisque les assises étaient sans fondement constitutionnel ? En réalité, dès les assises, quelques éléments permettaient de comprendre la nature de cette confiance accordée aux projets votés. En effet, à la veille des assises, un certain nombre de religieux s'inquiètent de ce que les textes votés ont des chances d'être repris et avalisés lors du chapitre provincial qui débute le lendemain: plusieurs textes sont alors rédigés qui manifestent des inquiétudes quant aux remises en cause de la vie dominicaine puis soumis aux signatures afin de prévenir toute entorse au droit dominicain. Ils permettent, dans leur contenu, de mesurer la distance qui est faite alors, dans une partie de la province, entre la confiance de principe accordée aux responsables afin qu'ils mènent les réformes nécessaires dans les charges qu'ils occupent (très peu s'y opposent) et une confiance accordée sur le fond qui prendrait appui sur 
les débats des assises et les textes votés pour leur donner une traduction par trop novatrice, voire subversive au moment du chapitre provincial. Parmi les protestataires, beaucoup mettent en avant la nécessité de mener les réformes dans la fidélité aux traditions et aux constitutions dominicaines. Certains brandissent la menace, voilée, d'un éclatement de la Province en plusieurs tendances. Dans un texte signé par plusieurs religieux venant des couvents dits de ministère et entrés pour la plupart dans l'Ordre dans l'entre-deux-guerres se manifestent des préventions à l'égard de bouleversements imposés au nom d'un nécessaire "pluralisme » :

Dans l'hypothèse où les Assises de l'Arbresle se termineraient par une déclaration (qu'aurait à entériner le Chapitre des jours suivants) sur le «pluralisme » des formes de vie dominicaine dans le monde d'aujourd'hui, les frères soussignés demandent [...] de vouloir bien prendre en considération la demande suivante: [...] qu'aux frères qui n'entrent pas dans cette perspective de vie religieuse dominicaine [...] soit accordée la possibilité de se regrouper - entre eux - selon des formes de "vie évangélique " tenant compte de leurs charismes particuliers. Ces divers regroupements seraient alors sous la juridiction du Provincial et de son Conseil et pourraient éventuellement donner naissance à une vice-province ${ }^{22}$.

Dans les dernières heures des assises, certains dominicains présents manifestent à nouveau une inquiétude quant à l'usage dévoyé qui pourrait être fait des textes votés lors du chapitre provincial qui débute le lendemain : des textes sont à nouveau mis en circulation, alors que certains des participants ont déjà quitté les lieux, et soumis aux signatures afin de protester par avance contre toute interprétation excessive des votes des assises par les pères capitulaires à qui ils sont adressés ${ }^{23}$. Les assises de la Tourette de 1969 ne peuvent être lues comme une assemblée révolutionnaire et les votes massifs ne doivent ni tromper sur leur sens ni masquer les clivages, notamment générationnels, qui traversent la province. En effet, elles ont davantage été vécues, à s'en tenir aux documents à la disposition, sur le mode finalement habituel de la participation aux instances dominicaines et particulièrement pour une assemblée qui, de droit, était consultative: confiance sur le fond aux personnes en charge, implication de principe dans les projets, volonté que les institutions ne soient pas mises en cause.

\section{Le Centre Thomas More : un projet en rupture avec les traditions de la Province de Lyon?}

S'il a été peu question, finalement, autant aux assises qu'au chapitre provincial qui les a suivies, du devenir du "campus de la Tourette", c'est bien parce que les enjeux ont été situés à un niveau autre que celui des réalisations concrètes. Il en va, dans les textes manifestant l'inquiétude de certains religieux avant et

22. Archives du couvent de la Tourette, carton Assises de la Tourette 1969. D’après les dates qui accompagnent les signatures, cette lettre collective peut être datée du début de la fin du mois de mai ou du début du mois de juin 1969. Une douzaine au moins de religieux l'a signée. D'autres courriers, individuels, expriment des inquiétudes similaires.

23. Ces textes sont conservés aux archives provinciales (ADPL B 1132). 
après la tenue des assises, de l'identité dominicaine telle qu'elle peut se vivre dans la Province de Lyon. De ce point de vue, la fondation des centres de rencontres et particulièrement de celui voué aux sessions de sciences humaines, ne peut s'inscrire, du moins au premier abord, qu'en rupture avec les traditions provinciales. Rappelons que la Province de Lyon est née en 1862 d'une rupture avec les conceptions lacordairiennes de la vie dominicaine, jugées trop libérales et qu'elle a construit son identité au miroir de l'intransigeantisme romain et dans la volonté d'une application sans nuances des observances dominicaines: une province de moines, plus qu'une province de dominicains selon ses détracteurs. Cette tradition est jalousement conservée par les responsables et formateurs successifs au fil des générations, au moins jusqu'au retour au studium savoyard de Saint-Alban-Leysse, après la période des expulsions, au début des années 1930. Dans les années 1950 encore, les traditions observantes sont défendues par une fraction de la province, en particulier celle des couvents de ministère et du couvent de noviciat (Angers ou Poitiers font figure de conservatoire des traditions observantes). Le couvent de Le Corbusier lui-même conserve encore certaines traces discrètes de la rigueur de cette vie dominicaine, recodée sous les atours de la modernité architecturale. Certains courriers envoyés aux organisateurs des assises ne manquent pas de rappeler la prégnance de cette tradition chez une partie des religieux.

Et pourtant, comme cela a été rappelé dans le bilan historiographique liminaire, la Province de Lyon est également celle dans laquelle la novation la plus étonnante a su éclore avec des réalisations comme celles de Jeunesse de l'Église ou d'Économie et Humanisme ou l'appel, pour la conception du nouveau couvent d'études, à un architecte emblématique d'une forme de modernité. Tout se passe comme si, à partir des années d'après-guerre surtout, se juxtaposaient dans la province des couvents encore très traditionnels et des entreprises novatrices: un mouvement de différenciation interne à la Province de Lyon s'est instauré sans qu'il remette en cause, au jugement des acteurs, son unité fondamentale, peut-être parce que celle-ci n'est jamais directement mise en question. De ce point de vue qui demanderait à être confirmé par des études complémentaires, la reconversion du couvent d'études de la Tourette et la naissance du Centre Thomas More, finalement, s'inscrivent dans la continuité d'une province qui s'est adaptée à son temps sans pour autant réaliser un quelconque aggiornamento et en laissant coexister en son sein des modèles différents.

\section{Le Centre Thomas More : un reniement de la théologie au profit des sciences humaines?}

Lors du Mai 68 des étudiants à la Tourette, le rejet de la théologie, ou du moins d'une certaine forme de théologie, par les étudiants contestataires semble faire consensus: Claude Pont en fait état dans ses souvenirs (Pont, 2010: 105-130); des documents l'illustrent ${ }^{24}$. Sans entrer dans un quelconque récit des événements qui se déroulent alors à la Tourette, et pour se tenir strictement à la question de

24. Ils sont classés dans un dossier relatif à Mai 1968 (ADPL B 1375 II). Les citations qui suivent proviennent toutes de ce dossier. 
la place de la théologie telle qu'elle peut alors être débattue, il est intéressant d'évoquer l'échange qui a lieu le 24 mai 1968 entre l'une des figures de la province, le théologien Christian Duquoc, et les étudiants réunis en assemblée autour du thème du «rôle du théologien ». Duquoc, dans un exposé liminaire, regrette que l'enseignement théologique soit suivi par les étudiants uniquement comme une contrainte: "Beaucoup d'étudiants en théologie choisissent d'être prêtres, ont une vocation pastorale... mais pour eux la théologie est un à-côté, une nécessité qui est imposée ». Les questions et remarques des étudiants à la suite de cette présentation convergent dans le sens d'une disqualification de la théologie et, peut-être plus encore, du théologien ${ }^{25}$. Les interventions de Jean-Yves Jolif, personnalité aussi centrale que mal connue du couvent, régent des études de 1961 à 1968, attisent les remises en cause des étudiants à l'endroit d'une théologie nostalgique de sa supériorité et de sa position de surplomb: «Il n'y a que les oisifs qui n'ont pas de métier!", s'exclame-t-il ${ }^{26}$. Les quelques récits disponibles de ces journées de contestation soulignent l'ascendant de Jolif sur les étudiants en ce mois de mai, au point que nombre de débats tournent autour d'un supposé " pouvoir jolifien » ou d'une «idéologie jolifienne », laquelle se caractériserait comme une opposition à la métaphysique et à la théologie au profit de la philosophie et d'un philo-marxisme, voire d'une anthropologie.

Sans aller plus loin dans l'analyse de ces événements, il convient de rappeler qu'à la différence des provinces de France et de Toulouse, la province de Lyon n'a jamais prétendu incarner une "école de théologie », qu'elle soit lue au prisme de l'histoire (Chenu, 1985) ou selon les intuitions maritainiennes, comme c'est le cas à Saint-Maximin puis à Toulouse autour de la Revue thomiste (Fouilloux, 1998: 99-140). Ses théologiens ${ }^{27}$ ne sont d'ailleurs pas, à la différence des deux autres provinces françaises, sollicités lors du concile Vatican II. Dès la régence du père Hyacinthe Paissac (1948-1954), dont Jolif est le disciple, ce qui caractérise le couvent d'études de la province de Lyon, c'est la formation philosophique. Jolif, plus tard, y ajoute l'engagement politique, au mouvement de la Paix et comme compagnon de route du Parti communiste avant, finalement, d'y adhérer. Mai 68 au couvent d'études se traduit par le départ pour Lyon et la constitution en petites communautés de la plupart des étudiants de la Tourette, accompagnés par quelques lecteurs dont Jean-Yves Jolif qui est de ce fait largement absent des préparatifs du Centre Thomas More. Si un accord se manifeste, et les votes aux assises comme au chapitre le suggèrent, c'est pour considérer qu'une formation théologique spécifiquement dominicaine enracinée dans une tradition provinciale peu lisible en ce domaine peut être assurée par l'Institut catholique de Lyon pour l'essentiel, en lien avec des engagements apostoliques et associatifs.

25. Quelques réactions en témoignent: «la plupart du temps le théologien m’est apparu comme quelqu'un qui farfouillait dans les bouquins et qui de ce fait, n'avait pas le temps de faire autre chose, le temps de vivre en un mot..."; un autre étudiant: "comment un homme qui manque d'une certaine expérience humaine, un métier par exemple au sens courant, peut-il s'adresser à d'autres hommes qui sont à l'intérieur de la foi, mais qui sont plongés dans le monde actuel, qui est un monde de réalisations?".

26. Ou encore: «N'est-ce pas parce que le théologien rêve toujours, a toujours la nostalgie d'une théologie constituée?".

27. Lebret mis à part, mais c'est à titre d'expert plus que de théologien qu'il est sollicité. 
Il reste qu'un couvent d'études n'est pas seulement un lieu d'enseignement de la théologie. Il est aussi, dans la tradition dominicaine, un lieu d'élaboration collective et de recherche. Dans le projet de Paul Grandin et des lecteurs, élaboré avant les assises et soumis aux discussions à l'automne 1969, la troisième des quatre propositions allait dans le sens d'un maintien de cette tradition et de sa mise à l'épreuve par la confrontation résolue avec d'autres disciplines: elle prévoyait la création d'un «centre de colloques théologiques avec participations multidisciplinaires". $\mathrm{Ne}$ devait-elle pas avoir la préférence des lecteurs, d'autant que le soutien de Mgr Haubtmann était acquis? Elle est pourtant écartée au profit de l'option résolue en faveur d'un centre dédié aux rencontres des sciences humaines des religions.

Je fais ici l'hypothèse, pour expliquer cet apparent paradoxe, que la fondation du Centre Thomas More puis, dans la foulée et de manière complémentaire, du Centre Albert-le-Grand, par la même équipe constituante, sur le domaine de la Tourette, est bien le résultat, d'une part, de la rupture introduite par les événements de Mai 1968 et, d'autre part, de la décision consécutive d'amener les étudiants dans la métropole lyonnaise, conformément aux aspirations de certains formateurs (Jean-Yves Jolif, Claude Pont). Le maintien du corps professoral et du couvent d'études à la Tourette, plutôt que leur déplacement sur Lyon comme cela avait été envisagé dans l'un des documents soumis aux participants des assises de juillet 1969, exprime une autre manière de faire vivre la tradition intellectuelle dominicaine. À cet égard, le choix du projet le plus radical, celui d'un centre dédié uniquement aux sciences humaines des religions, peut-être lu de deux manières: d'une part, comme une façon de passer la théologie au crible des sciences humaines; d'autre part, comme une façon d'éviter à la théologie, lourdement contestée dans sa raison d'être, de l'être plus encore par une confrontation qui apparaît alors anticipée. Le choix du projet en apparence le plus radical peut alors être lu comme le meilleur moyen de placer la théologie hors d'atteinte, dans l'attente que les théologiens aient effectué leur recyclage en sciences humaines. Si l'on suit cette hypothèse, le Centre Thomas More est tout sauf un lieu de sortie de la religion. Un indice peut-être apporté à l'appui de cette interprétation : il est fréquent de mettre en avant les départs massifs des étudiants du studium dans les années 1968-70 et leur retour à une vie laïque. Pourtant, la reconstitution des itinéraires permet de souligner la complexité de ce processus de sortie: les étudiants et une partie de ceux qui les encadre partis sur Lyon dans des petites communautés rassemblent le gros des départs des années 1969 et suivantes alors que ceux qui se maintiennent à la Tourette (étudiants et professeurs) ne quittent l'Ordre que de manière exceptionnelle.

\section{Conclusion}

Il est admis que l'introduction de la pensée aristotélicienne dans l'Europe médiévale a conduit des penseurs, Thomas d'Aquin en premier lieu, non seulement à apporter une réponse théologienne à ce défi intellectuel sur le mode de la réfutation mais, de manière sans doute plus décisive, à repenser le statut même de la théologie dont la caractéristique de «science » sort renforcée. Dans les années 1968, mis en question dans leurs compétences non pas tant de théologiens au sens classique du terme, mais de scientifiques, les professeurs du studium de la Tourette ont, en dignes 
successeurs du docteur Angélique, fait le choix d'un apprentissage des nouvelles rationalités véhiculées par les sciences humaines dans l'espoir d'offrir à la théologie l'opportunité de nouveaux développements. Ils n'ont pas cessé, ce faisant, de faire œuvre de théologiens. Le prix à payer, car il y en avait un, aura été de prendre acte, au sein même d'un lieu de formation et d'élaboration religieuse qui avait pour vocation d'être à l'abri de telles remises en cause, d'un nouvel ordre des savoirs dans lequel le règne de la théologie est moins que jamais assuré.

\author{
Tangi Cavalin \\ CEMS/IMM/EHESS \\ tangi.cavalin@gmail.com
}

\title{
Bibliographie
}

Bortel Philippe, 1975, Lieux d'Église. L'Arbresle, Les Fontaines, Boquen, La Sainte-Baume, Lourdes, Saint-Michel-de-Cuxa, Taizé, Paris, Éditions du Seuil.

Cavalin Tangi, Viet-Depaule Nathalie, 2015, «Un couvent pour la "stricte observance". Les fondateurs du Saint-Nom de Jésus à Lyon ", in Gueullette J.-M. (dir.), Un passé recomposé. Fondation et construction du couvent dominicain de Lyon, 1856-1888, Lyon, LARHA/RESEA, p. 29-48.

Chantin Jean-Pierre, 2015, "Chéry Charles ", Dictionnaire biographique des frères prêcheurs [En ligne], Notices biographiques. URL: http://dominicains.revues.org/654.

Chatelan Olivier, 2015, "Turin Gabriel », Dictionnaire biographique des frères prêcheurs [En ligne], Notices biographiques. URL: http://dominicains.revues.org/1880.

Chenu Marie-Dominique, 1985, Une école de théologie. Le Saulchoir, avec les études de Giuseppe Alberigo, Étienne Fouilloux, Jean Ladrière, Jean-Pierre Jossua, Paris, Éditions du Cerf.

Cholvy Gérard, Hilaire Yves-Marie, 1988, Histoire religieuse de la France contemporaine, 1930-1988, tome III, Toulouse, Privat.

Desroche Henri, 1992, Mémoires d'un faiseur de livres, Paris, Lieu commun.

Donneaud Henry, 2009, «Les écoles de théologie dominicaine à l'heure de la crise. Entre essoufflement, révolution et renouveau ", in Avon D., Fourcade M. (dir.), Un nouvel âge de la théologie? 1965-1980, Paris, Karthala.

Donneaud Henry, Laffay Augustin, Montagnes Bernard, 2015, La province dominicaine de Toulouse (XIX'-XXe siècles), préface d'Étienne Fouilloux, Paris, Karthala.

DuCret Roland, 1984, "La découverte des sciences humaines ", Échanges, 186, p. 8-11.

-, 1997, «Henri Desroche et le Centre Thomas More», in Poulat É., Ravelet C. (dir.), Henri Desroche. Un passeur de frontières, Paris, L'Harmattan.

Fouilloux Étienne, 1998, Une Église en quête de liberté, Paris, Desclée de Brouwer.

-, 2015, «Lumière et vie (1951-2013) ", Dictionnaire biographique des frères prêcheurs [En ligne], Notice thématique, Médias. URL: http://dominicains.revues.org/829.

Fouilloux Étienne, Gugelot Frédéric (dir.), 2014, Jésuites français et sciences humaines (années 1960), Lyon, LARHA/RESEA.

KeCK Thierry, 2004, Jeunesse de l'Église, 1936-1955, Aux sources de la crise progressiste, Paris, Éditions Karthala.

-, 2015, "Montuclard Maurice ", Dictionnaire biographique des frères prêcheurs [En ligne], Notice biographique. URL: http://dominicains.revues.org/613

Laudouze André, 1990, Vingt années de débats. Le Centre Thomas More, 1970-1990, La Tourette, Centre Thomas More. 
Lebel Béatrice, 2015, Boquen. Entre utopie et révolution, 1965-1976, Rennes, Presses universitaires de Rennes.

Mary André, 2008, "Henri Desroche: un anthropologue aux Archives », Archives de sciences sociales des religions, 141, p. 167-175.

Pelletier Denis, 1996, Économie et Humanisme. De l'utopie communautaire au combat pour le Tiers-Monde, 1941-1966, Paris, Éditions du Cerf.

Pelletier Denis, Schlegel Jean-Louis (dir.), 2012, À la gauche du Christ. Les chrétiens de gauche en France de 1945 à nos jours, Paris, Éditions du Seuil.

Pont Claude, 2010, Faire Part. Chemin de traverse vers une parole désarmée, Lyon, Éditions Entrevues.

Poulat Émile, Ravelet Claude (dir.), 1997, Henri Desroche. Un passeur de frontières, Paris, L'Harmattan.

Raison du Cleuziou Yann, 2016, De la contemplation à la contestation. La politisation des dominicains de la Province de France (années 1940-1970), Paris, Belin.

Ramognino Nicole (éd.), 1991, L'Instituant, les savoirs et les orthodoxies. En souvenir de Maurice Montuclard, Aix-en-Provence, Publications de l'Université de Provence.

Rousseau Sabine, 2015, "Biot François », Dictionnaire biographique des frères prêcheurs [En ligne], Notice biographique. URL: http://dominicains.revues.org/145. 